\title{
INCIDENCE RELATIONS IN UNICOHERENT SPACES
}

\author{
BY
}

\section{A. H. STONE}

1. Introduction. This paper is concerned with what might be called the "incidence geometry" of a space-the relations between the numbers of connected components of sets and their frontiers, unions and intersections. These relations turn out to be related in a fundamental way to the "degree of multicoherence" $\left.{ }^{1}\right)$ of the space; and here we shall consider only the unicoherent case $\left({ }^{2}\right)$. The following sample theorems may indicate the scope of the paper. (We make the assumption-essential for geometrical theorems of the type to be considered-that the space is locally connected.)

(1) Unicoherence is customarily defined in terms of closed sets: an equivalent definition is obtained if we use open sets instead (Theorem $3, \S 4.1$ ).

(2) If (in a unicoherent space) the frontiers of two sets $A, B$ are disjoint, then $b_{0}(A \cap B)+b_{0}(A \cup B)=b_{0}(A)+b_{0}(B)$, where $b_{0}(X)$ denotes, roughly, the number of components of $X\left(^{3}\right)$ (a special case of Theorem $6 \mathrm{~b}, \S 6.7$ ).

(3) If (in a unicoherent space) the frontiers of two connected sets $A, B$ intersect, then $b_{0}(\bar{A} \cap \bar{B}) \leqq b_{0}(\operatorname{Fr}(A) \cap \operatorname{Fr}(B))$, a "best possible" inequality (a special case of Theorem 5, §5.1).

(4) If $A, B, C$ are three connected subsets of a unicoherent space, and if every two of them meet, but $A \cap B \cap C=0$, then every two of their frontiers must meet (a special case of Theorem 8, §8.1).

Two features of the work perhaps require comment. In the first place, the result quoted in (1) is, of course, just what one would expect if the space were normal; but it will be obtained without assuming normality. In fact, almost all the results to be considered are independent of separation axioms. Secondly, the point-sets considered in (2)-(4) need not be open or closed, but are arbitrary; and this again will be the case for most of the theorems which follow.

2. Preliminaries.

2.1. Notations. Capital letters will usually denote subsets of a fixed nonempty topological space $S$; small letters will usually denote points or nonnegative integers. The notation $A \supset B$ means that $B$ is a subset of $A$; equality is not excluded. The complement of $A$ (with respect to $S$ ) is written $S-A$ or

Presented to the Society, April 23, 1943, under the title Connectedness and coherence; received by the editors March 13, 1948.

(1) Cf. $[29$, p. 83$]$ and $[9$, p. 153$]$. Numbers in brackets refer to the bibliography at the end of the paper.

(2) For the definition, see 2.2 below. Many of the results can be generalized to multicoherent spaces, but different methods are required.

( $\left.{ }^{3}\right)$ For the precise definition, see 2.2 below. 
$\operatorname{Co}(A)$, and the empty set is written 0 . We use $A-B$ to denote $A \cap \operatorname{Co}(B)$, whether $B \subset A$ or not. $\bar{A}$, or $\mathrm{Cl}(A)$, is the closure of $A$, and we write $\operatorname{Ext}(A)$ $=$ exterior of $A=\mathrm{Co}(\bar{A})$, Int $(A)=$ interior of $A=\operatorname{Ext}(\operatorname{Co}(A))$, and $\operatorname{Fr}(A)$ $=$ frontier of $A=\mathrm{Cl}(A) \cap \mathrm{Cl}(\mathrm{Co}(A))$.

2.2. Connectedness, and so on. Two sets $H, K$ are said to be "mutually separated," or separated, for short, if $\bar{H} \cap K=0=H \cap \bar{K}$. A set $A$ will be said to be connected relative to $B\left(^{4}\right)$ if, whenever $H$ is a nonempty subset of $A$ such that $H$ and $A-H$ are separated (equivalently, such that $H$ is both open and closed relative to $A$ ), then $H$ and $B$ are not separated. (Thus $A$ is connected if and only if $A$ is connected relative to each of its points.) Clearly, if every component of $A$ meets $B$, then $A$ is connected relative to $B$. Further, if $A$ is connected relative to $B$, and $B C C$, then $A$ is connected relative to $C$.

We write $b_{0}(X)=$ (number of components of $\left.X\right)-1$ if this number is finite, and $b_{0}(X)=\infty$ otherwise $\left(^{5}\right)$. (Thus $b_{0}(0)=-1$.) Clearly:

(1) $b_{0}(X) \geqq n$ if and only if $X$ is expressible in the form $X=H_{1} \cup H_{2} \cup \ldots$ $\cup H_{n+1}$, where $H_{i} \neq 0$ and $H_{i}, H_{j}$ are separated $(1 \leqq i<j \leqq n+1)$.

(2) If $A$ is connected relative to $B, b_{0}(A \cup B) \leqq b_{0}(B)$.

$S$ is said to be unicoherent if it is connected and, whenever $A, B$ are closed connected sets such that $A \cup B=S, A \cap B$ is connected.

Throughout the paper, the space $S$ will be assumed (except where the contrary is explicitly stated) to be a nonempty, connected, locally connected topological space. (In addition, $S$ will be assumed to be unicoherent in $\S \S 5-8$.)

2.3. Frontier properties. The following well known properties are easy to verify, and will be taken for granted in what follows $\left({ }^{6}\right)$.

(1) $\operatorname{Fr}(A)=\operatorname{Fr}(\operatorname{Co}(A))$; if $A \subset B \subset \bar{A}, \operatorname{Fr}(B) \subset \operatorname{Fr}(A)$.

(2) If a connected set $X$ meets both $A$ and $\operatorname{Co}(A), X$ meets $\operatorname{Fr}(A)$. In particular ( $S$ being connected) $\mathrm{Fr}(A) \neq 0$ unless $A=0$ or $S$.

(3) $\operatorname{Fr}(A \cup B) \cup \operatorname{Fr}(A \cap B) \cup\{\operatorname{Fr}(A) \cap \operatorname{Fr}(B)\}=\operatorname{Fr}(A) \cup \operatorname{Fr}(B)$.

(4) If $C$ is a component of $A, \operatorname{Fr}(C) \subset \operatorname{Fr}(A)$. In particular, a component of an open set is always open (being disjoint from its frontier).

(5) For any collection of sets $\left\{A_{\lambda}\right\}, \operatorname{Fr}\left(\cup A_{\lambda}\right) \subset \mathrm{Cl}\left\{\cup \operatorname{Fr}\left(A_{\lambda}\right)\right\}$.

3. Some fundamental characterizations of unicoherence.

3.1. It is convenient to begin by collecting together a number of useful elementary point-set properties which are equivalent to unicoherence. Most of these equivalences are well known, at least for Peano spaces. As always, the space $S$ is assumed to be connected, locally connected, and nonempty.

First we need some definitions. A set whose complement is connected is

(4) This notion is related to, but distinct from, one introduced by W. L. Ayres, Concerning subsets of a continuous curve which can be connected through the complement of the continuous curve, Amer. J. Math. vol. 50 (1928) pp. 521-534.

(5) Cf. [9, p. 153 (footnote 2)].

(6) Properties (1), (2) and (3) hold in an arbitrary topological space; but (4) and (5) are equivalent to the local connectivity of $S$ (see [15]). 
called non-separating. A set which is both connected and non-separating will be called "simple." The importance of such sets arises from the fact that (1) every component of a non-separating set is simple [14, p. 214].

3.2 THEOREM 1. The following properties of $S$ are equivalent $\left({ }^{7}\right)$ :

(i) $S$ is unicoherent.

(ii) The frontier of every simple set is connected.

(ii)' The frontier of every open $\left(^{8}\right)$ simple set is connected.

(iii) If $C$ and $D$ are disjoint connected sets such that $\operatorname{Fr}(C) \subset \operatorname{Fr}(D)$, then Fr $(C)$ is connected:

(iii)' If $C$ and $D$ are disjoint open connected sets such that $\operatorname{Fr}(C)=F$ $=\operatorname{Fr}(D)$, then $F$ is connected.

(iv) If $A$ and $B$ are open connected sets such that $\operatorname{Fr}(A) \cap \operatorname{Fr}(B)=0$, then $A \cap B$ is connected.

(v) If $M$ and $N$ are disjoint closed sets neither of which separates $\left({ }^{9}\right)$ two points $p, q$, then $M \cup N$ does not separate $p, q$.

(vi) If a closed set $A$ separates two points $p, q$, then so does some component of $A$.

Proof. The implications (iii) $\rightarrow$ (ii) $\rightarrow$ (ii)' are trivial. Hence it will suffice to prove (i) $\rightarrow$ (iii) and (ii) $\rightarrow$ (iii)' $\rightarrow$ (iv) $\rightarrow$ (v) $\rightarrow$ (vi) $\rightarrow$ (i).

(i) $\rightarrow$ (iii). For if $C$ and $D$ are as in (iii), let $\left\{E_{\lambda}\right\}$ be the set of components of $\operatorname{Co}(C \cup D)$; we have $\operatorname{Fr}\left(E_{\lambda}\right) \subset \operatorname{Fr}(C \cup D) \subset \operatorname{Fr}(C) \cup \operatorname{Fr}(D)=\operatorname{Fr}(D)$, and therefore (unless $C=0=D$ ) each $\bar{E}_{\lambda}$ meets $\bar{D}$. Thus, on defining $X=\bar{D} \cup \cup \bar{E}_{\lambda}$, we have that $X$ is connected. But clearly $\mathrm{Co}(C) \subset X \subset \mathrm{Cl}(\mathrm{Co}(C)$ ); thus $\mathrm{Cl}(X)=\mathrm{Cl}(\mathrm{Co}(C))$. The sets $\mathrm{Cl}(C)$ and $\mathrm{Cl}(\mathrm{Co}(C))$ are closed and connected, and they cover $S$; hence by, (i), their intersection $\operatorname{Fr}(C)$ is connected.

We note for later use that if $C$ is open here, $\operatorname{Co}(C)=X$, so that $C$ is simple.

(ii)' $\rightarrow$ (iii)'. Let $C$ and $D$ satisfy the requirements of (iii)'; they then, a fortiori, satisfy those of (iii), so that (as just noted) $C$ is now simple. Hence $\operatorname{Fr}(C)$ is connected, by (ii)'.

(iii)' $\rightarrow$ (iv). Suppose (iv) is false; then there exist open connected sets $A$, $B$, with disjoint frontiers, such that $A \cap B$ is not connected. Let $C$ be one component of $A \cap B$; then $C$ is closed relative to $A \cap B$, so that, on selecting any point $x \in(A \cap B)-C$, we have $x \notin \bar{C}$. Let $D$ be the component of $\operatorname{Co}(\bar{C})$ which contains $x$; thus $D$ is open. The connected open set $C$ is disjoint from $D$, and thus from $\bar{D}$; let $E$ be the component of $\operatorname{Co}(\bar{D})$ which contains $C$. It is easy to see that $\operatorname{Fr}(D)=\operatorname{Fr}(E)$, so that from (iii)' we have that $\operatorname{Fr}(D)$ is

( $\left.{ }^{7}\right)$ The equivalence of (i), (ii), (v) and (vi) was proved by Kuratowski [16] for normal $S$. Property (iii) is due essentially to R. L. Moore (Fund. Math. vol. 6 (1924) p. 189). For Peano spaces, (v) can be considerably generalized; cf. [18], [10], [28]; and, in a different direction, some of the implications between these properties can also be generalized [12, Theorem 1]. For property (vi) in non-locally-connected spaces, see [3].

(8) Or, equivalently, closed (by complementation).

( $\left.{ }^{9}\right)$ "separates" $p, q$ if $\operatorname{Co}(X)=H \cup K$ where $H, K$ are separated and $p \in H, q \in K$. 
connected. But $\operatorname{Fr}(D) \subset \operatorname{Fr}(C) \subset \operatorname{Fr}(A \cup B) \subset \operatorname{Fr}(A) \cup \operatorname{Fr}(B)$, a union of two disjoint closed sets. Hence $\operatorname{Fr}(D)$ is contained in one of them, say in $\operatorname{Fr}(A)$. The connected set $A$ meets both $D$ (in $x$, at least) and $\operatorname{Co}(D)$ (in $C$, at least), and therefore meets $\operatorname{Fr}(D)$. That is, $A$ meets $\operatorname{Fr}(A)$, which is impossible because $A$ is open.

(iv) $\rightarrow$ (v). Let $M, N$ be disjoint closed sets, neither of which separates $p, q$. We can of course assume that neither $p$ nor $q$ belongs to $M \cup N$. Then $p, q$ must belong to the same component $A$ of $\operatorname{Co}(M)$, and to the same component $B$ of $\operatorname{Co}(N)$; and since $\operatorname{Fr}(A) \cap \operatorname{Fr}(B) \subset M \cap N=0$, (iv) shows that $A \cap B$ is connected. Thus $p, q$ belong to a connected subset of $\operatorname{Co}(M \cup N)$, and so cannot be separated by $M \cup N$.

$(\mathrm{v}) \rightarrow($ vi). Let $A$ be a closed set separating two points $p, q$. The component $D$ of $\operatorname{Co}(A)$ which contains $p$ cannot contain $q$; and since $\operatorname{Fr}(D)$ $\subset \operatorname{Fr}(A) \subset A$, we have $q \notin \bar{D}$. Let $C$ be the component of $\operatorname{Co}(\bar{D})$ which contains $q$; thus $C, D$ are disjoint open connected sets, and $\operatorname{Fr}(C) \subset \operatorname{Fr}(D)$. We shall show that $\operatorname{Fr}(C)$ is connected. For if not, we have $\operatorname{Fr}(C)=M \cup N$ where $M$, $N$ are disjoint nonempty closed sets. The set $C \cup N \cup D$ is a connected set containing both $p$ and $q$, and disjoint from $M$; hence $M$ does not separate $p$ and $q$. Similarly, neither does $N$. Hence, from (v), neither does $\operatorname{Fr}(C)$, which is absurd. As a connected subset of $A, \operatorname{Fr}(C)$ is contained in some component $K$ of $A$. Clearly $K$ separates $p, q$, as required.

(vi) $\rightarrow$ (i). Suppose $A$ and $B$ are closed connected sets such that $A \cup B=S$ and $A \cap B$ is not connected; thus $A \cap B=H \cup K$, where $H, K$ are disjoint, closed and nonempty. We have:

(1) There exists a component $D$ of $\operatorname{Co}(A)$ such that $\operatorname{Fr}(D)$ is not connected.

For otherwise we have, for every component $D_{\lambda}$ of $\operatorname{Co}(A)$, either $\operatorname{Fr}(D) \subset H$ or $\operatorname{Fr}\left(D_{\lambda}\right) \subset K$. Define $H^{*}=H \cup \cup\left\{D_{\lambda} \mid \operatorname{Fr}\left(D_{\lambda}\right) \subset H\right\}, \quad K^{*}=K$ $\cup \cup\left\{D_{\lambda} \mid \operatorname{Fr}\left(D_{\lambda}\right) \subset K\right\}$. It is easy to verify that $H^{*}, K^{*}$ are disjoint closed sets such that $H^{*} \cup K^{*}=B$, contrary to the assumption that $B$ is connected.

As a component of a nonseparating set, $D$ is simple-that is, $\operatorname{Co}(D)$ is connected. It now follows, by a repetition of the argument used to prove (1), that there exists a component $C$ of $\operatorname{Co}(\bar{D})$ such that $\operatorname{Fr}(C)$ is not connected. Clearly $C$ and $D$ are open connected sets, and $\operatorname{Fr}(C) \subset \operatorname{Fr}(D)$. Pick any points $p \in C, q \in D$. From (vi), a component $K$ of $\operatorname{Fr}(C)$ must separate $p$ and $q$. Pick $x \in \operatorname{Fr}(C)-K$; the connected set $C \cup(x) \cup D$ contains both $p$ and $q$, and is disjoint from $K$-giving the desired contradiction.

3.3. Sufficient conditions. The properties just considered are not of ten easy to apply in deciding whether a given space is unicoherent. However, the more powerful criteria given by Borsuk and Cech, though not directly applicable to spaces of the present generality, can readily be generalized:

THEOREM 2. Let $S$ be a connected and locally arcwise connected nonempty Hausdorff space (satisfying the $T_{2}$ axiom of [2]). Then each of the following 
conditions is sufficient for $S$ to be unicoherent:

(a) Given any simple closed curve $J$ in $S$, there exists a set $T$ such that ( $\alpha$ ) $S \supset T \supset J,(\beta) T$ is normal, and $(\gamma) T$ cannot be retracted on $J$.

(b) The 1-dimensional Betti number of $S$, in the sense of finite continuous cycles with rational coefficients, is zero.

(c) Every simple closed curve in $S$ is contractible.

REMARKs. If $S$ is normal, the condition (a) is also necessary for unicoherence, and the theorem in this case reduces to that of Borsuk [4, p. 184]. Similarly, for polytopes at any rate, condition (b) is also necessary and reduces to a theorem of Borsuk and Cech ([5], [7]; cf. also [22]). Condition (c) can be regarded as generalizing an argument of Wilson, and is not necessary for unicoherence (failing, for example, for the projective plane) $\left({ }^{10}\right)$.

The proof for (a) is a straightforward generalization of that of Borsuk [4, pp. 184-187], except that 3.2 (iii)' furnishes a more convenient startingpoint; and the sufficiency of (b) and (c) follows easily.

3.4. EXAmples. From condition (c) of 3.3, we see at once that any convex subset of a linear topological space will be unicoherent. In particular, the Cartesian product $R^{\mathfrak{m}}$ of $\mathfrak{m}$ open intervals ( $\mathfrak{m}$ being any cardinal number) and the product $I^{\mathfrak{m}}$ of $\mathfrak{m}$ closed intervals are unicoherent. If $\mathfrak{m} \leqq \boldsymbol{N}_{0}$, this is well known; but if $\mathfrak{m}>\boldsymbol{N}_{0}$ the "classical" methods will not apply to these spaces, as $R^{\mathfrak{m}}$ is then not normal, and $I^{\mathfrak{m}}$, though normal, is not completely normal (see [21] and [23]).

Again, it is easy to see that the deformation required in $3.3(\mathrm{c})$ can be made to avoid any sufficiently "thin" set. For example, if $T$ is a linear topological space of (linear) dimension not less than 3 , then the complement in $T$ of any countable subset is connected, locally arcwise connected and unicoherent. The same holds for the complement of any separable subset of $R^{\mathfrak{m}}$ if $\mathrm{m}>c\left({ }^{11}\right)$.

A deeper result in this direction is the following: If $X$ is any subset of $R^{n}$ $(3 \leqq n<\infty)$ which contains no compact subset of dimension greater than $n-3$ (for example, $X$ of dimension not greater than $n-3$, or $X$ totally imperfect), then $R^{n}-X$ is unicoherent. If $X$ is closed, this follows from a theorem of Alexandroff $\left({ }^{12}\right)$; and the general case can be reduced to this one on using property (iv) of 3.2 .

Finally, a more "pathological" example, which will be useful for constructing counterexamples later, is the transfinite line, or $\alpha$-arc, $L_{\alpha}$, obtained from the ordinal numbers not exceeding a given ordinal $\alpha$ by intercalating intervals between successive ordinals and imposing the order-topology (cf. $[33$, p. 491$]) . L_{\alpha}$ is a connected, locally connected, compact $\left({ }^{13}\right)$ Hausdorff

(10) See [31]. Condition (c) is however equivalent to unicoherence for subsets of the plane, and in some other cases; cf. [6, Satz 6, p. 133].

(11) This fails when $\mathfrak{m}=c ; R^{c}$ is separable.

(12) [1]; cf. also [3, Theorem 25].

(13) That is, bicompact. 
space. Theorem 2 does not apply to $L_{\alpha}$, since $L_{\alpha}$ is not arcwise connected; but its unicoherence is immediate from the definition.

It can be proved, though with more trouble, that any Cartesian product of transfinite lines is unicoherent.

4. Equivalence of open and closed sets.

4.1. Let us provisionally define a space to be "open-unicoherent" if it satisfies the definition of unicoherence except that open connected sets are used instead of closed ones; that is, $S$ is open-unicoherent if it is connected and nonempty and satisfies: whenever $A, B$ are open connected sets such that $A \cup B=S$, then $A \cap B$ is connected.

One would expect this notion to coincide with unicoherence for normal spaces; the surprising thing is that this coincidence holds without any separation axioms. We have:

TheOREM 3. For connected, locally connected, topological spaces, unicoherence and open-unicoherence are equivalent.

4.2. Proof. One implication is easy. Let $S$ be unicoherent, and let $A, B$ be open connected sets such that $A \cup B=S$. Then since $\operatorname{Fr}(A) \cap A=0$ we have $\operatorname{Fr}(A) \subset B$ and therefore $\operatorname{Fr}(A) \cap \operatorname{Fr}(B)=0$. Thus $A \cap B$ is connected, from 3.2(iv). That is, unicoherence implies open-unicoherence.

The converse implication is more delicate (in the absence of normality). We prove it in a number of stages, after first discussing a generalization of the notion of connectedness.

4.3. Normal connectedness. We shall say that two sets $H, K$ are "normally separated" (relative to the containing space $S$ ) if they are separated by some closed set-that is, if there exist disjoint open sets $U, V$ in $S$ such that $U \supset H$ and $V \supset K$. A set $A$ is "normally connected" (14) (relative to $S$ ) if it is not expressible as the union of two non-empty normally separated sets. Thus a connected set is always normally connected, while conversely if $A$ is normally connected and either (i) $S$ is completely normal, or (ii) $A$ is closed and $S$ is normal, or (iii) $A$ is open (and $S$ is arbitrary), then $A$ is connected.

That normally connected sets need not be connected, even if $S$ is normal, compact, connected, locally connected and unicoherent, is shown by the following example. Take $S$ to be the product $I^{1} \times L_{\omega}$ (cf. 3.4), where $\omega$ is the first uncountable ordinal, and $I^{1}$ can be regarded as the closed interval $(0,1)$. Let $p$ denote the point $(1, \omega)$, and define $H=\left(1 \times L_{\omega}\right)-(p), K=\left(I^{1} \times \omega\right)-(p)$. It is easy to see that $H$ and $K$, though separated, are not normally separated relative to $S$. Since clearly they are connected, the set $H \cup K$ is normally connected (rel. $S$ ) but not connected.

Many of the elementary properties of connected sets can be generalized immediately to normally connected sets $\left({ }^{15}\right)$. In particular, one readily verifies

(14) This term has been used before, with a different meaning [30]; however, no confusion is likely to result.

(15) In fact, "normal separation" makes $S$ a "separation space" in the sense of [25]. 
the following two properties (the first of which holds even if $S$ is not locally connected).

(1) A necessary and sufficient condition that $A$ be normally connected is that, whenever $A$ meets both $X$ and $\operatorname{Co}(X), A$ also meets $\operatorname{Fr}(X)$.

(2) A necessary and sufficient condition that $A$ be normally connected is that, given any open set $U \supset A$, there exists a connected set $B$ such that $A \subset B \subset U$.

4.4. Returning to the proof that open-unicoherence implies unicoherence, we now show:

LEMMA. If $S$ is open-unicoherent, then

(a) the frontier of every simple set is normally connected.

Let $A$ be simple (that is, $A$ and $\operatorname{Co}(A)$ both connected), and let $U$ be an open set containing $\operatorname{Fr}(A)$. Let $B$ be the union of all components of $U$ which meet $\operatorname{Fr}(A)$; then $B$ is open, and $\operatorname{Fr}(A) \subset B \subset U$. Further, $B$ is connected relative to $A$ (cf. 2.2), so that $A \cup B$ is connected (2.2(2)). Similarly $\operatorname{Co}(A) \cup B$ is connected. The sets $A \cup B$ and $\operatorname{Co}(A) \cup B$ are easily seen to be open; hence their intersection, $B$, is connected; and from 4.3(2) this implies that $\operatorname{Fr}(A)$ is normally connected.

4.5. Lemma. Property (a) (of 4.4) implies:

(b) If $A$ and $B$ are simple subsets of $S$ such that $\operatorname{Fr}(A) \cap \operatorname{Fr}(B)=0$, then either $A \subset B$, or $B \subset A$, or $A \cap B=0$, or $A \cup B=S$. (Cf. [17, p. 153].)

Suppose not. Then $A$ meets both $B$ and $\operatorname{Co}(B)$, and so meets $\operatorname{Fr}(B)$. It follows that $\operatorname{Fr}(B) \subset A$, since otherwise $\operatorname{Fr}(B)$ must meet $\operatorname{Fr}(A)$ (from (a) and 4.3(1)), contrary to hypothesis. Again, we may replace $A$ by $\operatorname{Co}(A)$ in this argument, and obtain $\operatorname{Fr}(B) \subset \operatorname{Co}(A)$. Thus $\operatorname{Fr}(B)=0$, which is impossible.

4.6. Lemma. 'Property (b) (of 4.5) implies:

(c) If $A$ and $B$ are intersecting connected open sets with disjoint frontiers, there exist connected open sets $A^{*} \supset A, B^{*} \supset B$, such that $A^{*} \cap B^{*}=A \cap B$ and $A^{*} \cup B^{*}=S$.

Let $C, D$ be any components of $\operatorname{Co}(A), \operatorname{Co}(B)$ respectively. Then $C$ and $D$ are simple sets $(3.1(1))$ with disjoint frontiers, so that property (b) gives

$$
C \subset D, \text { or } C \supset D \text {, or } C \cap D=0 \text {, }
$$

the alternative $C \cup D=S$ being excluded here by the fact that $A \cap B \neq 0$. Now define $E=$ union of all components $C$ of $\operatorname{Co}(A)$ which are contained in $\mathrm{Co}(B)$, and $F=$ union of those components $D$ of $\mathrm{Co}(B)$ which are contained in $\operatorname{Co}(A \cup E)$. Write $A^{*}=A \cup E, B^{*}=B \cup F$. On using (1) it is easy to verify that $A^{*}$ and $B^{*}$ have the desired properties.

4.7. If $S$ is open-unicoherent, then $S$ is unicoherent. 
Let $A$ and $B$ be any intersecting open subsets of $S$ with disjoint frontiers. Since $S$ has property (c), there exist connected open sets $A^{*} \supset A, B^{*} \supset B$, such that $A^{*} \cap B^{*}=A \cap B$ and $A^{*} \cup B^{*}=S$. From the open-unicoherence of $S$ it follows that $A^{*} \cap B^{*}$ is connected; that is, $A \cap B$ is connected. From property (iv) of Theorem 1 (3.2), $S$ is unicoherent. This completes the proof of Theorem 3.

4.8. An extension property. The property (c) of 4.6 can now be strengthened to the following theorem, which will be useful later:

Theorem 4. Suppose $S$ is unicoherent. Then:

(d) Given any sets $A, B$ such that $A \cup B$ is connected and $\operatorname{Fr}(A) \cap \operatorname{Fr}(B)$ $\cap \operatorname{Fr}(A \cup B)=0$, there exist sets $A^{*}, B^{*}$ with the following properties: $A^{*} \supset A$, $B^{*} \supset B, A^{*} \cup B^{*}=S, A^{*} \cap B^{*}=A \cap B, \operatorname{Fr}\left(A^{*}\right) \subset \operatorname{Fr}(A), \operatorname{Fr}\left(B^{*}\right) \subset \operatorname{Fr}(B), A^{*}$ is connected relative to $A$ (cf. 2.2), $B^{*}$ is connected relative to $B$, and further $A^{*}$ and $B^{*}$ are closed if $A$ and $B$ are closed, and open if $A$ and $B$ are open.

Proof. Let $\left\{C_{\lambda}\right\}$ be the components of $\operatorname{Co}(A \cup B)$; they are simple, so (3.2(ii)) $\operatorname{Fr}\left(C_{\lambda}\right)$ is connected. Since $\operatorname{Fr}\left(C_{\lambda}\right) \subset \operatorname{Fr}(A \cup B) \subset \operatorname{Fr}(A) \cup \operatorname{Fr}(B)$, and $\operatorname{Fr}\left(C_{\lambda}\right) \cap \operatorname{Fr}(A) \cap \operatorname{Fr}(B)=0$, we have that, for each $\lambda$, either $\operatorname{Fr}\left(C_{\lambda}\right)$ $C \operatorname{Fr}(A)$ or $\operatorname{Fr}\left(C_{\lambda}\right) \subset \operatorname{Fr}(B)$. Define $A^{*}=A \cup \cup\left\{C_{\lambda} \mid \operatorname{Fr}\left(C_{\lambda}\right) \subset \operatorname{Fr}(A)\right\}$, $B^{*}=B \cup \cup\left\{C_{\lambda} \mid \operatorname{Fr}\left(C_{\lambda}\right) \subset \operatorname{Fr}(B)\right\}$. It is easy to verify that $A^{*}$ and $B^{*}$ satisfy all the requirements.

REMARK. $A^{*}$ and $B^{*}$ are not uniquely determined by the properties listed in (d), in general.

4.9. Equivalences. If $S$ is normal, each of properties (a), (b), (c), (d) (4.4-4.8) is equivalent to unicoherence. This is trivial for (a); for the others, it follows from the following somewhat strengthened form of a theorem of Wallace [26]: If $S$ is normal and not unicoherent, there exist three open connected sets $G_{1}, G_{2}, G_{3}$ such that $\cup G_{i}=S, \cap \bar{G}_{i}=0, G_{i} \cap G_{j} \neq 0$ and $\operatorname{Fr}\left(G_{i}\right) \cap \operatorname{Fr}\left(G_{j}\right)=0$ $(i \neq j)$. (We omit the proof.) If now $S$ is normal and not unicoherent, it is easy to see that (c) fails when $A=G_{1}, B=G_{2}$; and (b) and (d) fail a fortiori.

However, for non-normal $S$, it is worth remarking that none of the properties considered in this section (except, of course, open-unicoherence) is equivalent to unicoherence, in general. This is shown by the following example. Let $S$ be any infinite set of points, and select any two infinite subsets $X, Y$ such that $X \cup Y=S$ and $X \cap Y$ consists of exactly two points. $S$ is topologized by taking the closed sets to be all sets of the form $F, X \cup F, Y \cup F$, $S$, where $F$ runs over all finite sets (including 0 ). It is easy to see that $S$ is a compact, connected, locally connected $T_{1}$ space and that (a) and (d) hold in it-so that (b) and (c) must hold too. But clearly $S$ is not unicoherent.

The same example shows the need for normality (or at least for some separation axiom stronger than $T_{1}$ ) in the theorem of Wallace stated above.

The hypothesis of local connectedness is, as usual, essential for the theorems just considered; for example, it is easy to construct plane continua 
which are open-unicoherent but not unicoherent. It would, however, be interesting to know whether the implication "unicoherence-open-unicoherence" holds without local connectedness.

4.10. Convention. Throughout the rest of the paper, the space $S$ will be assumed to be unicoherent, as well as nonempty, connected, and locally connected.

5. Intersections of two connected sets and their frontiers.

5.1. We have seen (3.2(iv)) that if $A$ and $B$ are connected open sets with disjoint frontiers, then (if $S$ is unicoherent) $A \cap B$ is connected. We now investigate the case in which $\operatorname{Fr}(A) \cap \operatorname{Fr}(B) \neq 0$. The most natural conjecture would probably be

$$
b_{0}(A \cap B) \leqq b_{0}\{\operatorname{Fr}(A) \cap \operatorname{Fr}(B)\}
$$

but this is easily seen to be false in general (even in $R^{3}$ ), though it holds in the 2 -sphere (see 7.5 below). However, (1) becomes true if $A$ and $B$, instead of being open, are closed. We prove a little more than this:

TheOREM 5. If $A$ and $B$ are any subsets of $S$ such that $\bar{A}$ and $\bar{B}$ are connected and $\operatorname{Fr}(A) \cap \operatorname{Fr}(B) \neq 0$, then

$$
b_{0}(\bar{A} \cap \bar{B}) \leqq b_{0}\{\operatorname{Fr}(A) \cap \operatorname{Fr}(B)\} .
$$

The proof is divided into two cases, depending on whether $b_{0}(\bar{A} \cap \bar{B})$ is finite or not; curiously enough, it is the infinite case which gives the more trouble. We recall that the space $S$ is henceforth assumed unicoherent.

5.2. LEMmA. If $A$ and $B$ are connected sets with intersecting frontiers, and if $C$ is a component of $A \cap B$ which is open relative to $A \cap B$, then $\operatorname{Fr}(A) \cap \operatorname{Fr}(B)$ $\cap \operatorname{Fr}(C) \neq 0$.

Suppose not. Let $p \in \operatorname{Fr}(A) \cap \operatorname{Fr}(B)$; thus $p \notin \operatorname{Fr}(C)$, whence $p \notin C$. Let $D$ be the component of $\operatorname{Co}(C)$ which contains $p . D$ is simple, and so $\operatorname{Fr}(D)$ is connected (3.2(ii)). But it is easy to see that $\operatorname{Fr}(D) \subset \operatorname{Fr}(A) \cup \operatorname{Fr}(B)$, and that $\operatorname{Fr}(D) \cap \operatorname{Fr}(A) \cap \operatorname{Fr}(B)=0$; hence there is no loss of generality in assuming $\operatorname{Fr}(D) \subset \operatorname{Fr}(A)$, and $\operatorname{Fr}(D) \cap \operatorname{Fr}(B)=0$. Hence $\operatorname{Fr}(D) \subset \operatorname{Int}(B)$, and accordingly $A \cap \operatorname{Fr}(D) \subset A \cap \bar{C} \cap B=C$, since $C$ is closed relative to $A \cap B$. It is easy to deduce that the sets $A \cap D$ and $A-D$ are separated.

Now we have $p \in \operatorname{Fr}(A) \cap \operatorname{Int}(D)$, which shows that $A \cap D \neq 0$; and $A-D \supset C \neq 0$. Thus $A$ is not connected-a contradiction.

CoROLlARY 1. If $A$ and $B$ are connected sets with intersecting frontiers, and if either (i) $A$ and $B$ are both open, or (ii) $b_{0}(A \cap B)<\infty$, then $A \cap B$ is connected relative to $\operatorname{Fr}(A) \cap \operatorname{Fr}(B)$.

CoRollary 2. If $A$ and $B$ are connected intersecting sets and if either (i) $A$ and $B$ are both open, or (ii) $b_{0}(A \cap B)<\infty$, then $\operatorname{Fr}(A) \cap \operatorname{Fr}(B) \cap \operatorname{Fr}(A \cap B)$ 
$=0$ implies $\operatorname{Fr}(A) \cap \operatorname{Fr}(B)=0$.

REMARK. It is easy to see that the restrictions (i) and (ii) cannot both be omitted in Corollary 1 , even if $A$ and $B$ are closed and $S$ is the plane. On the other hand, it can be shown that they are superfluous in Corollary 2, at any rate provided $S$ is normal.

\subsection{Lemma. Theorem 5 holds if $b_{0}(\bar{A} \cap \bar{B})<\infty$.}

Let $\bar{A}$ and $\bar{B}$ be connected, and let $\operatorname{Fr}(A) \cap \operatorname{Fr}(B) \neq 0$. If $\operatorname{Fr}(\bar{A}) \cap \operatorname{Fr}(\bar{B})$ $=0$, an application of Theorem 4 (4.8) to the sets $\bar{A}$ and $\bar{B}$ shows that $\bar{A} \cap \bar{B}$ is connected; thus Theorem 5 holds in this case. If $\operatorname{Fr}(\bar{A}) \cap \operatorname{Fr}(\bar{B}) \neq 0$, Corollary 1 of 5.2 shows that $\bar{A} \cap \bar{B}$ is connected relative to $\operatorname{Fr}(A) \cap \operatorname{Fr}(B)$; thus, from 2.2(2), $b_{0}(\bar{A} \cap \bar{B}) \leqq b_{0}\{\operatorname{Fr}(A) \cap \operatorname{Fr}(B)\}$, as required.

5.4. Lemma. Let $A$ and $B$ be connected sets, and let $F$ be the union of all the components of $\operatorname{Fr}(A)$ which meet $B$. Then $(A \cap B) \cup F$ is connected $\left({ }^{16}\right)$.

Suppose not; thus $(A \cap B) \cup F=H \cup K$, where $H$ and $K$ are nonempty separated sets. If $A \cap B=0, B$ is contained in a unique component $C$ of $\operatorname{Co}(A) ; \operatorname{Fr}(C)$ is connected (from 3.2(ii)), and it is easy to see that $F$ is either empty or is the unique component of $\operatorname{Fr}(A)$ which contains $C$. Thus this case can be eliminated, and we may assume $A \cap B \neq 0$.

Now let $\left\{C_{\lambda}\right\}$ be the collection of those components of $\operatorname{Co}(A)$ which meet $B$. Being connected, $B$ must meet each $\operatorname{Fr}\left(C_{\lambda}\right)$; and $\operatorname{Fr}\left(C_{\lambda}\right)$ is connected (3.2(ii)) and is a subset of $\operatorname{Fr}(A)$. Hence $\operatorname{Fr}\left(C_{\lambda}\right) \subset F \subset H \cup K$. Thus, for each $\lambda$, either $\operatorname{Fr}\left(C_{\lambda}\right) \subset H$ or $\operatorname{Fr}\left(C_{\lambda}\right) \subset K$. Define $H^{*}=H \cup \cup\left\{C_{\lambda} \mid \operatorname{Fr}\left(C_{\lambda}\right) \subset H\right\}$, $K^{*}=K \cup \cup\left\{C_{\lambda} \mid \operatorname{Fr}\left(C_{\lambda}\right) \subset K\right\}$. Clearly $H^{*} \cup K^{*} \supset B \cup F$, and $H^{*} \cap K^{*}=0$. It is not hard to verify that $H^{*}$ and $K^{*}$ are in fact separated. Hence $B \cup F$ is not connected. But $F$ is connected relative to the connected set $B$, so that $B \cup F$ must be connected (cf. 2.2(2)); and this contradiction proves the lemma.

5.5. The proof of Theorem 5 can now be completed. In view of 5.3 , we can assume $b_{0}(\bar{A} \cap \bar{B})=\infty$, and must show that $b_{0}\{\operatorname{Fr}(A) \cap \operatorname{Fr}(B)\}=\infty$.

Define $E=$ union of the components of $\operatorname{Fr}(A)$ which meet both $\bar{B}$ and $\operatorname{Co}(\bar{B}), F_{1}=$ union of the components of $\operatorname{Fr}(A)$ which meet $\bar{B}$, and $F_{2}=$ union of the components of $\operatorname{Fr}(\bar{A})$ which meet $\bar{B}$. Thus $F_{1} \supset F_{2}$, and we have $(\bar{A} \cap \bar{B}) \cup E=(\bar{A} \cap \bar{B}) \cup F_{1}$. But clearly $F_{1}$ is connected relative to $\bar{A} \cap \bar{B}$, and 5.4 shows that $(\bar{A} \cap \bar{B}) \cup F_{2}$ is connected; hence so is $(\bar{A} \cap \bar{B}) \cup F_{2} \cup F_{1}$ $=(\bar{A} \cap \bar{B}) \cup E$.

If $b_{0}(E)=\infty$, then, since each component of $E$ contains at least one component of $\operatorname{Fr}(A) \cap \operatorname{Fr}(B)$, we have $b_{0}\{\operatorname{Fr}(A) \cap \operatorname{Fr}(B)\}=\infty$, as required. Thus we may assume that $E$ has only a finite number of components, say $E_{1}, E_{2}, \cdots, E_{n}$. Hence $E$ is closed.

On the other hand, $b_{0}(E \cap \bar{B})=\infty$. For otherwise $E$ could meet only a

${ }^{(16)}$ This lemma is closely related to several results of R. L. Moore; see [19]. 
finite number, say $m$, of components of $\bar{A} \cap \bar{B}$. From 2.2(1) we can write $\bar{A} \cap \bar{B}=H_{1} \cup H_{2} \cup \ldots \cup H_{m+1}$ where the sets $H_{j}$ are closed, nonempty and pairwise disjoint; and, for some $j, H_{j}$ would be disjoint from $E$-contradicting the fact that $(\bar{A} \cap \bar{B}) \cup E$ is connected. Thus for some $i$ we have $b_{0}\left(E_{i} \cap \bar{B}\right)$ $=\infty, E_{i}$ being a component of $E$ and therefore of $\operatorname{Fr}(A)$. Accordingly we can write, for every $k, E_{i} \cap \bar{B}=K_{1} \cup K_{2} \cup \ldots \cup K_{k}$, a union of $k$ closed, nonempty, pairwise disjoint sets. Since $E_{i}$ is connected, there exists for each $p(1 \leqq p \leqq k)$ a point $x_{p} \in K_{p} \cap \mathrm{Cl}\left(E_{i}-K_{p}\right)$; and it readily follows that $x_{p} \in \operatorname{Fr}(B)$. Thus the sets $K_{p} \cap \operatorname{Fr}(B)$ are nonempty; and it follows that $b_{0}\left\{E_{i} \cap \operatorname{Fr}(B)\right\} \geqq k-1$. A fortiori, $b_{0}\{\operatorname{Fr}(A) \cap \operatorname{Fr}(B)\} \geqq k-1$ for every $k$; and so, finally, $b_{0}\{\operatorname{Fr}(A) \cap \operatorname{Fr}(B)\}=\infty$.

5.6. Conversely, Theorem 5 implies unicoherence, at least for normal $T_{1}$ spaces. In fact, if $R$ is a nonempty connected, locally connected, normal $T_{1}$ space which is not unicoherent, it is easy to construct two open connected subsets $A, B$ of $R$ such that (i) $\operatorname{Fr}(A) \cap \operatorname{Fr}(B)$ consists of a single point, (ii) $\bar{A} \cap \bar{B}$ has at least two components. But for nonnormal spaces Theorem 5 may hold without the space being unicoherent, as is shown by the example given in 4.9 .

\section{Sets with disjoint frontiers.}

6.1. We return now to the consideration of sets with disjoint frontiers. The main results (stated in 6.7 below) will be easy consequences of the following theorem, which is analogous to the Meyer-Vietoris addition theorem.

TheOREM 6. If $A$ and $B$ are any sets such that $\operatorname{Fr}(A) \cap \operatorname{Fr}(B)=0$, then $b_{0}(A \cap B)+b_{0}(A \cup B)=b_{0}(A)+b_{0}(B)\left({ }^{17}\right)$.

(The space $S$, of course, is assumed to be unicoherent.)

We first show that this holds with $\geqq$ replacing $=$, under much more general circumstances; the proof of the reverse inequality will then be carried out in several steps, starting with the special case (which contains the main difficulties of the proof) in which $A$ and $B$ are connected.

6.2. Lemma. Let $A$ and $B$ be arbitrary sets such that the sets $A-B$ and $B-A$ are separated. Then

$$
b_{0}(A \cap B)+b_{0}(A \cup B) \geqq b_{0}(A)+b_{0}(B)\left({ }^{18}\right) .
$$

Let $m, n$ be any integers such that $b_{0}(A) \geqq m \geqq 0$ and $b_{0}(B) \geqq n \geqq 0$. (We can clearly assume that neither $A$ nor $B$ is empty.) It will suffice to prove that $b_{0}(A \cap B)+b_{0}(A \cup B) \geqq m+n$.

We can write $A=\cup A_{i}(1 \leqq i \leqq m+1)$ and $B=\cup B_{j}(1 \leqq j \leqq n+1)$, where every two of the sets $A_{i}$, and every two of the sets $B_{j}$, are separated, and

(17) This generalizes both Theorem 1 (iv) (3.2) and a theorem of Knaster [13, Lemma 4].

(18) This is a slight generalization of a theorem announced by Whyburn [27]; the case in which $A \cup_{B}$ and $A \cap B$ are connected is given in [14, p. 211]. The condition that $A-B$ and $B-A$ be separated was introduced by Wallace [25]. 
where $A_{i} \neq 0 \neq B_{j}$. It is easy to verify that $A_{i}-B_{j}$ and $B_{j}-A_{i}$ are separated, for every $i$ and $j$; hence $A_{i}$ and $B_{j}$ are separated whenever they are disjoint.

Form a linear graph $G$ by taking $m+n+2$ vertices $a_{i}, b_{j}(1 \leqq i \leqq m+1$, $1 \leqq j \leqq n+1)$ and joining $a_{i}, b_{j}$ by a 1 -cell if and only if the "corresponding" sets $A_{i}, B_{j}$ intersect. (That is, $G$ is the 1 -dimensional skeleton of the nerve of the sets $A_{i}, B_{j}$.) Let $G$ have $k$ components $G_{s}(1 \leqq s \leqq k)$, and define $C_{s}=$ union of those sets $A_{i}, B_{j}$ whose corresponding vertices are in $G_{s}$. It readily follows that the sets $C_{8}$ are pairwise separated; thus, since $\cup C_{s}=A \cup B$, we have $b_{0}(A \cup B) \geqq k-1$.

But, by the Euler-Poincaré theorem for graphs, $G$ has at least $(m+n+2)-k$ edges, each of which corresponds to a distinct nonempty intersection of the form $A_{i} \cap B_{j}=D_{t}$, say $(1 \leqq t \leqq m+n+2-k)$. Two distinct sets $D_{t}$ are clearly separated, and $\cup D_{t}=A \cap B$; hence $b_{0}(A \cap B) \geqq m+n+1-k$, and the result follows.

REMARK 1. The condition that $A-B$ and $B-A$ be separated is implied by each of the following:

(1) $A$ and $B$ both closed, or both open, relative to $A \cup B$ (in particular, $A$ and $B$ both closed, or both open, in $S)$;

(2) $A$ and $B$ separated;

(3) $\mathrm{Co}(A)-\mathrm{Co}(B)$ and $\operatorname{Co}(B)-\operatorname{Co}(A)$ separated;

(4) $\operatorname{Fr}(A) \cap \operatorname{Fr}(B)=0$.

REMARK 2. This lemma and its proof are valid in an arbitrary topological space.

6.3. LemmA. If $A$ and $B$ are connected sets with disjoint frontiers, then $A \cap B$ is connected.

If $A$ and $B$ are both open, this has been proved already (Theorem 1 (iv), 3.2). Following an idea of $\mathrm{C}$. Chevalley, we first show that it remains true if only $A$ is open, and then deduce it in general.

Suppose, then, that $A$ is open and that $A \cap B$ is not connected; thus $A \cap B$ $=H \cup K$, where $H$ and $K$ are nonempty and separated.

Let $C$ be any component of $\operatorname{Co}(A)$ which meets $B$. Since $B$ is connected, $B$ meets $\operatorname{Fr}(C)$; hence, since $\operatorname{Fr}(C)$ is a connected subset of $\operatorname{Fr}(A)$ (from 3.2(ii)) and is therefore disjoint from $\operatorname{Fr}(B)$, we have $\operatorname{Fr}(C) \subset \operatorname{Int}(B)$. Let $D$ be the component of $\operatorname{Int}(B)$ which contains $\operatorname{Fr}(C)$; thus $D$ is connected and open. Further, $\operatorname{Fr}(D) \subset \operatorname{Fr}(B)$, so $\operatorname{Fr}(A) \cap \operatorname{Fr}(D)=0$. Hence, by the case of the lemma in which both sets are open, $A \cap D$ is connected.

(It will be important for the subsequent arguments that this is the only use which will be made of the openness of $A$.)

Since $A \cap D$ is a connected subset of $A \cap B=H \cup K$, we have either $A \cap D \subset H$ and $A \cap D \cap K=0$, or the same with $H$ and $K$ interchanged. It readily follows that either $\operatorname{Fr}(C) \subset \bar{H}$ and $\operatorname{Fr}(C) \cap \bar{K}=0$, or $\operatorname{Fr}(C) \subset \bar{K}$ and $\operatorname{Fr}(C) \cap \bar{H}=0$. 
Now let $\left\{C_{\lambda}\right\}$ be the collection of all the components of $\operatorname{Co}(A)$ which meet $B$. Define $H^{*}=\bar{H} \cup \cup\left\{C_{\lambda} \mid \operatorname{Fr}\left(C_{\lambda}\right) \subset \bar{H}\right\}, K^{*}=\bar{K} \cup \cup\left\{C_{\lambda} \mid \operatorname{Fr}\left(C_{\lambda}\right) \subset \bar{K}\right\}$. From 2.3(5) we see that $H^{*}$ and $K^{*}$ are closed, while clearly $H^{*} \cup K^{*} \supset B$, $B \cap H^{*} \supset H \neq 0$, and $B \cap K^{*} \supset K \neq 0$. Thus, since $B$ is connected, there must exist a point $x \in B \cap H^{*} \cap K^{*}$. It readily follows that $x \in A \cap B \cap \bar{H} \cap \bar{K}=H \cap K$, since $H$ and $K$ are closed relative to $A \cap B$; and this is impossible. This establishes the lemma if $A$ is open.

Finally, in the general case, we repeat the above argument. It applies unchanged, provided that we can justify the conclusion that if $A$ and $D$ are connected sets with disjoint frontiers, of which $D$ is open, then $A \cap D$ is connected; for this is the only step in which the openness of $A$ was used above. And this justification is now provided by the case of the lemma in which one of the sets is open-that is, by the case established above. Thus the proof is complete.

6.4. Lemma. Let $A$ and $B$ be sets with disjoint frontiers, $B$ being connected, and let $A_{1}, A_{2}$ be different components of $A$, both of which meet $B$. Then $A_{1}-B$ and $A_{2}-B$ are disconnected by $A \cap B\left({ }^{19}\right)$.

It will evidently suffice to prove this assuming that $A_{1}$ and $A_{2}$ are the only components of $A$. Then $A \cup B$ is connected, so Theorem 4 (4.8) gives the existence of sets $A^{*}, B^{*}$ such that $A^{*} \supset A, B^{*} \supset B, A^{*} \cup B^{*}=S, A^{*} \cap B^{*}$ $=A \cap B, A^{*}$ is connected relative to $A, B^{*}$ is connected, $\operatorname{Fr}\left(A^{*}\right) \subset \operatorname{Fr}(A)$, and $\operatorname{Fr}\left(B^{*}\right) \subset \operatorname{Fr}(B)$.

If the lemma is false, there will exist a connected set $C$ meeting $A_{1}-B$ and $A_{2}-B$ but not $A \cap B$. There are two cases.

(1) If $C \subset A^{*}$, then $A^{*}$, being connected relative to the connected subset $A_{1} \cup C \cup A_{2}$ of $A^{*}$, is connected (2.2(2)). Since $\operatorname{Fr}\left(A^{*}\right) \cap \operatorname{Fr}\left(B^{*}\right) \subset \operatorname{Fr}(A)$ $\cap \operatorname{Fr}(B)=0$, the previous lemma (6.3) shows that $A^{*} \cap B^{*}$ is connected. But this is impossible, since $A^{*} \cap B^{*}=A \cap B=\left(A_{1} \cap B\right) \cup\left(A_{2} \cap B\right)$, a union of two nonempty separated sets.

(2) If $C \nsubseteq A^{*}$, then $C$ meets $B^{*}-A^{*}$, and also meets $A^{*}-B^{*}$ (in $A_{i}-B$ at least). Further, $C \subset S-(A \cap B)=\left(A^{*} \cup B^{*}\right)-\left(A^{*} \cap B^{*}\right)=\left(A^{*}-B^{*}\right)$ $\cup\left(B^{*}-A^{*}\right)$; and the sets $A^{*}-B^{*}$ and $B^{*}-A^{*}$ are separated (cf. 6.2, Remark 1 (4)). Thus $C$ cannot be connected, and again we have a contradiction.

6.5. Proof of Theorem 6. Let $A$ and $B$ be any sets with disjoint frontiers. In view of 6.2 , it will suffice to prove that $b_{0}(A \cup B)+b_{0}(A \cap B) \leqq b_{0}(A)$ $+b_{0}(B)$, and we may clearly assume that the right-hand side is finite. Let $b_{0}(A)=m-1, b_{0}(B)=n-1$; the proof will go by double induction over the non-negative integers $m, n$. The desired result is trivial if $m$ or $n$ is 0 , and has been proved in 6.3 if $m=n=1$; thus we may assume $n \geqq 2$, and that the theorem holds for all $m^{\prime} \leqq m$ and $n^{\prime}<n$.

${ }^{(19)}$ We shall say that $X$ "disconnects" $P$ and $Q$ if no connected subset of $\operatorname{Co}(X)$ can meet both $P$ and $Q$. 
Let the components of $A$ and $B$ be $A_{i}(1 \leqq i \leqq m)$ and $B_{j}(1 \leqq j \leqq n)$ respectively, and define $B^{\prime}=B-B_{n}$. The hypothesis of induction then gives.

$$
b_{0}\left(A \cup B^{\prime}\right)+b_{0}\left(A \cap B^{\prime}\right) \leqq m+n-3 .
$$

Suppose $B_{n}$ meets exactly $r$ components of $A(r \geqq 0)$, which we may take to be $A_{1}, A_{2}, \cdots, A_{r}$. We shall show that no two of $A_{1}, \cdots, A_{r}$ can belong to the same component of $A \cup B^{\prime}$. For otherwise (as is easy to see, for example, on considering the "nerve" of the sets) there exists a finite sequence of the form

$$
A_{i}, B_{q_{1}}, A_{p_{1}}, B_{q_{2}}, \cdots, B_{q_{x}}, A_{j} \quad\left(i, j \leqq r ; q_{1}, \cdots, q_{x}<n\right)
$$

each member of which meets the next. Choose such a sequence having as few members as possible; for this sequence we have $p_{1}, p_{2}, \cdots$, all greater than $r$. Thus, on defining $D=B_{q_{1}} \cup A_{p_{1}} \cup B_{q_{2}} \cup \cdots \cup B_{q_{x}}$, we have that $D$ is a connected subset of $\mathrm{Co}\left(B_{n}\right)$ which meets both $A_{i}-B_{n}$ and $A_{j}-B_{n}$. But, from 6.4, $A \cap B_{n}$ disconnects $A_{i}-B_{n}$ and $A_{j}-B_{n}$, giving a contradiction.

Thus, if $C_{1}, C_{2}, \cdots, C_{t}$ are the components of $A \cup B^{\prime}$, we may choose the notation so that $A_{i} \subset C_{i}(1 \leqq i \leqq r)$. The components of $A \cup B$ are evidently $\left(C_{1} \cup C_{2} \cup \cdots \cup C_{r} \cup B_{n}\right), C_{r+1}, C_{r+2}, \cdots, C_{t}$, so that $b_{0}(A \cup B)=b_{0}\left(A \cup B^{\prime}\right)$ $-r+1$. Again, $A \cap B=\left(A \cap B^{\prime}\right) \cup\left(A_{1} \cap B_{n}\right) \cup\left(A_{2} \cap B_{n}\right) \cup \ldots \cup\left(A_{r} \cap B_{n}\right)$, where (from 6.3) each of the sets $A_{i} \cap B_{n}$ is connected. Hence $b_{0}(A \cap B)$ $\leqq b_{0}\left(A \cap B^{\prime}\right)+r$. On combining these results, we obtain $b_{0}(A \cup B)+b_{0}(A \cap B)$ $\leqq m+n-2$, as desired.

6.6. $A$ modification. If the sets $A$ and $B$ are closed, the hypothesis that $\operatorname{Fr}(A) \cap \operatorname{Fr}(B)=0$ can be weakened; and as this result will be useful later, we state it.

TheOREM 6a. If $A$ and $B$ are closed sets such that $\operatorname{Fr}(A) \cap \operatorname{Fr}(B)$ $\cap F r(A \cup B)=0$, then $b_{0}(A \cap B)+b_{0}(A \cup B)=b_{0}(A)+b_{0}(B)$.

The proof of this is very similar to that of Theorem 6 . The first step is to establish it when the sets $A$ and $B$ are connected (cf. 6.3); and here this follows immediately from Theorem 4 (4.8). The analogue of the lemma in 6.4 goes as before, under the (harmless) extra hypothesis that $b_{0}(A)<\infty$; and the extension to the general case requires only trivial changes in the argument of 6.5 .

Corollary. For any set $X, \quad b_{0}(\operatorname{Fr}(X))=b_{0}(\mathrm{Cl}(X))+b_{0}(\mathrm{Cl}(\mathrm{Co}(X)))$.

(This generalizes Theorem 1(ii), 3.2.)

6.7. Extension to $n$ sets. Given any finite collection of set $A_{1}, A_{2}, \cdots, A_{n}$, we define the "elementary symmetric sets" $X_{1}=\mathrm{U} A_{i}, X_{r}=\mathrm{U}\left(A_{i_{1}} \cap A_{i_{2}} \cap \ldots\right.$ $\cap A_{i_{r}}$ ) (where $1 \leqq i_{1}<i_{2}<\cdots<i_{r} \leqq n$ ), and finally $X_{n}=\bigcap A_{i}$. The results obtained in this section can all be generalized, as follows: 
Theorem 6b. If $A_{i}-A_{j}$ and $A_{j}-A_{i}$ are separated $(1 \leqq i<j \leqq n)$, then $\sum_{1}^{n} b_{0}\left(A_{i}\right) \leqq \sum_{1}^{n} b_{0}\left(X_{r}\right)$. If $\operatorname{Fr}\left(A_{i}\right) \cap \operatorname{Fr}\left(A_{j}\right)=0$, or if the sets $A_{i}$ are closed and $\operatorname{Fr}\left(A_{i}\right) \cap \operatorname{Fr}\left(A_{j}\right) \cap \operatorname{Fr}\left(A_{i} \cup A_{j}\right)=0$, then $\sum_{1}^{n} b_{0}\left(A_{i}\right)=\sum_{1}^{n} b_{0}\left(X_{r}\right)$.

This follows by a straightforward induction over $n$, the results considered previously corresponding to the case $n=2$. The first part of the theorem (cf. 6.2) holds in an arbitrary topological space.

\section{Index formulae for arbitrary sets.}

7.1. Notation. Let $A_{1}, A_{2}, \cdots, A_{n}$ be any subsets of $S$, not necessarily distinct. As in 6.7 we define $X_{r}(1 \leqq r \leqq n)$ to be the set of those points which belong to $A_{i}$ for $r$ or more values of $i$. Where it is necessary to emphasize its dependence on the sets $A_{i}$, we write $X_{r}$ as $X_{r}\left(A_{1}, \cdots, A_{n}\right)$. We write

$$
h\left(A_{1}, \cdots, A_{n}\right)=\sum_{1}^{n} b_{0}\left(X_{r}\right)-\sum_{1}^{n} b_{0}\left(A_{i}\right),
$$

provided all the numbers on the right are finite. To deal with sets having infinitely many components, we make the convention that statements (of equality or inequality) involving the $h$-function are to be regarded as statements about $b_{0}$ in which negative terms are transposed to the opposite sides. With this convention, for example, the first part of Theorem $6 \mathrm{~b}(6.7)$ is completely equivalent to:

(2) $\quad h\left(A_{1}, \cdots, A_{n}\right) \geqq 0$ provided $A_{i}-A_{j}$ and $A_{j}-A_{i}$ are separated $(1 \leqq i<j \leqq n)$.

Similarly the other other parts of Theorem $6 \mathrm{~b}$ simply give sufficient conditions for $h\left(A_{1}, \cdots, A_{n}\right)=0$.

7.2. Some identities. Now let $A_{1}, \cdots, A_{n}, B_{1}, \cdots, B_{n}$ be sets such that $A_{i} \cup B_{i}=S(1 \leqq i \leqq n)$, and write $X_{r}\left(A_{1}, \cdots, A_{n}\right)=P_{r}, X_{r}\left(B_{1}, \cdots, B_{n}\right)=Q_{r}$. It is easy to verify that

$$
P_{r} \cup Q_{n+1-r}=S
$$

and that

(2)

$$
\begin{array}{r}
X_{r}\left(P_{1} \cap Q_{n}, P_{2} \cap Q_{n-1}, \cdots, P_{n} \cap Q_{1}\right)=\cup\left\{\left(P_{i} \cap Q_{j}\right) \mid i+j=n+r\right\} \\
=X_{r}\left(A_{1} \cap B_{1}, A_{2} \cap B_{2}, \cdots, A_{n} \cap B_{n}\right) .
\end{array}
$$

As an immediate consequence of (2), we have identically

$$
\begin{array}{r}
h\left(A_{1}, \cdots, A_{n}\right)+h\left(B_{1}, \cdots, B_{n}\right)+h\left(P_{1} \cap Q_{n}, P_{2} \cap Q_{n-1}, \cdots, P_{n} \cap Q_{1}\right) \\
+\sum_{1}^{n} h\left(P_{i}, Q_{n+1-i}\right)=h\left(A_{1} \cap B_{1}, \cdots, A_{n} \cap B_{n}\right)+\sum_{1}^{n} h\left(A_{i}, B_{i}\right) .
\end{array}
$$

(Properties (1)-(3) are, of course, valid in an arbitrary space.)

7.3. To apply the preceding to a unicoherent space $S$, let $E_{1}, \cdots, E_{n}$ be 
arbitrary subsets of $S$, and take $A_{i}=\bar{E}_{i}, B_{i}=\mathrm{Cl}\left(\mathrm{Co}\left(E_{i}\right)\right)$. From Theorem 6a (6.6) we have now $h\left(A_{i}, B_{i}\right)=0=h\left(P_{i}, Q_{n+1-i}\right)$ (in view of 7.2(1)); thus, provided that $h\left(A_{i}, B_{i}\right)$ and $h\left(P_{i}, Q_{n+1-i}\right)$ are all defined, 7.2(3) gives:

Theorem 7. $h\left(\bar{E}_{1}, \cdots, \bar{E}_{n}\right)+h\left\{\mathrm{Cl}\left(\mathrm{Co}\left(E_{1}\right)\right), \cdots, \mathrm{Cl}\left(\mathrm{Co}\left(E_{n}\right)\right)\right\}+h\left(P_{1}\right.$ $\left.\cap Q_{n}, \cdots, P_{n} \cap Q_{1}\right)=h\left\{\operatorname{Fr}\left(E_{1}\right), \cdots, \operatorname{Fr}\left(E_{n}\right)\right\}$, where $P_{r}=X_{r}\left(\bar{E}_{1}, \cdots, \bar{E}_{n}\right)$ and $Q_{8}=X_{s}\left(\mathrm{Cl}\left(\mathrm{Co}\left(E_{1}\right)\right), \cdots, \mathrm{Cl}\left(\mathrm{Co}\left(E_{n}\right)\right)\right)$.

Finally, the above proviso can be removed, since if it fails we have either $b_{0}\left(\operatorname{Fr}\left(E_{i}\right)\right)=\infty$ or $b_{0}\left(P_{i} \cap Q_{n+1-i}\right)=\infty$, and so (from 7.1(2)) the theorem asserts merely $\infty=\infty$.

Corollary. $h\left(\bar{E}_{1}, \cdots, \bar{E}_{n}\right) \leqq h\left\{\operatorname{Fr}\left(E_{1}\right), \cdots, \operatorname{Fr}\left(E_{n}\right)\right\}$.

For if $h\left\{\mathrm{Cl}\left(\mathrm{Co}\left(E_{1}\right)\right), \cdots, \mathrm{Cl}\left(\mathrm{Co}\left(E_{n}\right)\right)\right\}$ and $h\left(P_{1} \cap Q_{n}, \cdots, P_{n} \cap Q_{1}\right)$ are both defined, this follows immediately from the theorem on applying 7.1(2). If $h\left(P_{1} \cap Q_{n}, \cdots, P_{n} \cap Q_{1}\right)$ is defined but $h\left\{\mathrm{Cl}\left(\mathrm{Co}\left(E_{1}\right)\right), \cdots\right.$, $\left.\mathrm{Cl}\left(\mathrm{Co}\left(E_{n}\right)\right)\right\}$ is not defined, the corollary still follows immediately unless $b_{0}\left(\operatorname{Cl}\left(\operatorname{Co}\left(E_{i}\right)\right)\right)=\infty$ for some $i$; but in this case we have $b_{0}\left(\operatorname{Fr}\left(E_{i}\right)\right)=\infty$ (6.6, Corollary), and, from 7.1(2), the corollary now asserts $\infty=\infty$. Finally, if $h\left(P_{1} \cap Q_{n}, \cdots, P_{n} \cap Q_{1}\right)$ is not defined, 7.2(2) shows that $h\left\{\operatorname{Fr}\left(E_{1}\right), \cdots\right.$, $\left.\operatorname{Fr}\left(E_{n}\right)\right\}$ is not defined either, so that (in accordance with the convention introduced in 7.1) the corollary asserts merely that some quantity is not greater than $\infty$, and so holds in all cases.

In a sense, the above corollary to Theorem 7 is a generalization of Theorem 5 (5.1). In fact, if we apply it with $n=2$ to sets $E_{1}, E_{2}$ which have connected closures and intersecting frontiers (so that $b_{0}\left\{\operatorname{Fr}\left(E_{1}\right) \cup \operatorname{Fr}\left(E_{2}\right)\right\} \leqq b_{0}\left(\operatorname{Fr}\left(E_{1}\right)\right.$ ) $\left.+b_{0}\left(\operatorname{Fr}\left(E_{2}\right)\right)\right)$, we readily obtain the assertion of Theorem $5-b_{0}\left(\bar{E}_{1} \cap \bar{E}_{2}\right)$ $\leqq b_{0}\left\{\operatorname{Fr}\left(E_{1}\right) \cap \operatorname{Fr}\left(E_{2}\right)\right\}$-provided that $b_{0}\left(\mathrm{Cl}\left(\mathrm{Co}\left(E_{1}\right)\right)\right)+b_{0}\left(\mathrm{Cl}\left(\mathrm{Co}\left(E_{2}\right)\right)\right)$ is finite. Unfortunately, this proviso is not easy to remove.

7.4. It is of interest to compare Theorem 7, Corollary with the following property, which holds in an arbitrary topological space (and can likewise be deduced from 7.2(3)): For any sets $E_{1}, \cdots, E_{n}$,

(1) $\begin{aligned} \sum_{1}^{n} b_{0}\left\{X_{r}\left(\bar{E}_{1}, \cdots, \bar{E}_{n}\right)\right\}+ & \sum_{1}^{n} b_{0}\left\{X_{r}\left(\operatorname{Cl}\left(\operatorname{Co}\left(E_{1}\right)\right), \cdots, \mathrm{Cl}\left(\operatorname{Co}\left(E_{n}\right)\right)\right)\right\} \\ & \leqq \sum_{1}^{n} b_{0}\left\{X_{r}\left(\operatorname{Fr}\left(E_{1}\right), \cdots, \operatorname{Fr}\left(E_{n}\right)\right)\right\}+n b_{0}(S) .\end{aligned}$

In particular, if $S$ is connected, and $E_{1}, E_{2}$ are proper subsets of $S$, we obtain

(2) $b_{0}\left(\bar{E}_{1} \cap \bar{E}_{2}\right)+b_{0}\left(\bar{E}_{1} \cup \bar{E}_{2}\right)$

$$
\leqq b_{0}\left\{\operatorname{Fr}\left(E_{1}\right) \cap \operatorname{Fr}\left(E_{2}\right)\right\}+b_{0}\left\{\operatorname{Fr}\left(E_{1}\right) \cup \operatorname{Fr}\left(E_{2}\right)\right\} .
$$

7.5. Application to $S^{2}$. Now take $S$ to be the 2 -sphere $S^{2}$. We have: 
TheOREM 7a. For any subsets $E_{1}, \cdots, E_{n}$ of $S^{2}$,

$$
h\left\{\operatorname{Int}\left(E_{1}\right), \cdots, \operatorname{Int}\left(E_{n}\right)\right\} \leqq h\left\{\operatorname{Fr}\left(E_{1}\right), \cdots, \operatorname{Fr}\left(E_{n}\right)\right\} .
$$

For a fundamental theorem of Straszewicz ([24]; cf. also [8, p. 101]) asserts, in effect, that for two closed subsets $F_{1}, F_{2}$ of $S^{2}$ we have $h\left(F_{1}, F_{2}\right)$ $=h\left\{\mathrm{Co}\left(F_{1}\right), \operatorname{Co}\left(F_{2}\right)\right\}$. An easy induction argument extends this result to $n$ closed sets; thus $h\left\{\operatorname{Int}\left(E_{1}\right), \cdots, \operatorname{Int}\left(E_{n}\right)\right\}=h\left\{\mathrm{Cl}\left(\operatorname{Co}\left(E_{1}\right)\right), \cdots\right.$, $\left.\mathrm{Cl}\left(\mathrm{Co}\left(E_{n}\right)\right)\right\}$, and the result follows from Theorem 7, Corollary (7.3).

COROLLARY. If $E_{1}$ and $E_{2}$ are connected open sets in $S^{2}$, and if their frontiers intersect, then

$$
b_{0}\left(E_{1} \cap E_{2}\right) \leqq b_{0}\left\{\operatorname{Fr}\left(E_{1}\right) \cap \operatorname{Fr}\left(E_{2}\right)\right\}\left({ }^{20}\right) .
$$

If $b_{0}\left(\operatorname{Co}\left(E_{1}\right)\right)+b_{0}\left(\operatorname{Co}\left(E_{2}\right)\right)<\infty$, this follows readily from the case $n=2$ of Theorem $7 \mathrm{a}$; the general case can be reduced to this one.

8. Systems of connected sets and their frontiers.

8.1. Theorem 8. Let $A_{1}, A_{2}, \cdots, A_{n}$ be $n$ connected sets, every $n-1$ of which, but not all of which, have a point in common; and suppose $n \geqq 3$. Then at least $2 n-3$ pairs of their frontiers meet. (More precisely, there will be at least $2 n-3$ pairs of numbers $i, j$, with $1 \leqq i<j \leqq n$, such that $\left.\operatorname{Fr}\left(A_{i}\right) \cap \operatorname{Fr}\left(A_{j}\right) \neq 0\left({ }^{21}\right).\right)$

REMARK. It is easy to see that the number $2 n-3$ is "best possible" for each $n$, even if the sets $A_{i}$ are open (or closed) and simple and $S$ is the plane. (Of course, the space $S$ is assumed to be unicoherent.) Further, the case $n=3$ of the theorem is already characteristic of unicoherence, for normal spaces at least; this is an immediate consequence of the theorem of Wallace quoted in 4.9 .

The proof requires a graph-theoretical lemma, which we proceed to derive.

8.2. Let $p$ be any vertex of a (finite) linear graph $G$. $A$ vertex $q$ is said to be "adjacent" to $p$ if $p$ and $q$ are the end points of a 1-cell (edge) of $G$. It will be assumed that $G$ is "proper"-that is, that no vertex is adjacent to itself. The "shell" of a vertex $p$ in $G$ is defined to be the subgraph of $G$ consisting of (i) all vertices adjacent to $p$, and (ii) all 1-cells of $G$ both of whose end points are adjacent to $p$.

We write $\alpha_{0}(G)$ and $\alpha_{1}(G)$ for the numbers of vertices and 1-cells of $G$, respectively.

LEMмA. If $G$ is connected, and if the shells of all its vertices are connected, then $\alpha_{1}(G) \geqq 2 \alpha_{0}(G)-3$.

This is proved by induction over $\alpha_{0}(G)$. We can assume $G$ has a vertex $p$

(20) Compare 5.1(1). Some particular cases are given in [20, p. 109].

(21) If $n=3$, a slightly stronger result can be deduced from Theorem $4(4.8) ; \operatorname{Fr}\left(A_{i}\right) \cap \operatorname{Fr}\left(A_{j}\right)$ $\Phi_{A_{i}} \cap A_{j} \cap \operatorname{Int}\left(A_{i} \cup A_{j}\right)(1 \leqq i<j \leqq 3)$. It is not known whether an analogous sharpening of the theorem holds for $n>3$. 
adjacent to at most 3 others; for otherwise a simple counting argument gives $\alpha_{1}(G) \geqq 2 \alpha_{0}(G)$. If the shell of $p$ consists of 3 vertices and 21 -cells, say $q_{1} q_{2}$ and $q_{2} q_{3}$, we form a new graph $H$ by omitting $p$ and the 1 -cells $p q_{i}(i=1,2,3)$ from $G$, and adjoining a new 1-cell $q_{1} q_{3}$. In all other cases we form $H$ from $G$ by merely omitting $p$ and the 1 -cells incident with $p$. The reader will readily verify that $H$ satisfies the conditions which were imposed on $G$; hence $\alpha_{1}(H) \geqq 2 \alpha_{0}(H)-3$, and the result follows.

8.3. Returning to the proof of Theorem 8 , we define $B_{1}=$ union of those components of $\mathrm{Co}\left(A_{1}\right)$ which are not contained in $\operatorname{Co}\left(A_{2}\right) \cup \cdots \cup \operatorname{Co}\left(A_{n}\right)$, $B_{2}=$ union of those components of $\mathrm{Co}\left(A_{2}\right)$ which are not contained in $B_{1} \cup \operatorname{Co}\left(A_{3}\right) \cup \cdots \cup \operatorname{Co}\left(A_{n}\right)$, and generally $B_{i}=$ union of those components of $\operatorname{Co}\left(A_{i}\right)$ which are not contained in $B_{1} \cup B_{2} \cup \ldots \cup B_{i-1} \cup \operatorname{Co}\left(A_{i+1}\right)$ $\cup \ldots \cup \operatorname{Co}\left(A_{n}\right)$. The sets $B_{i}(1 \leqq i \leqq n)$ are easily seen to be nonseparating (cf. 3.1); and clearly $B_{i} \subset \mathrm{Co}\left(A_{i}\right)$ and

$$
\operatorname{Fr}\left(B_{i}\right) \subset \operatorname{Fr}\left(A_{i}\right) .
$$

By construction, no component of any $B_{i}$ can be contained in $\cup\left\{B_{j} \mid j \neq i\right\}$. And since $\cap A_{i}=0$, while every $n-1$ of the sets $A_{i}$ have a common point, we see that $S$ is the union of all $n$, but of no $n-1$, of the sets $B_{i}$. Thus the sets $B_{i}$ certainly have the following properties:

(2) $B_{1}, \cdots, B_{n}$ are $n(\geqq 3)$ nonempty nonseparating sets; $\cup B_{i}=S$; $B_{i} \cup B_{j} \neq S$; and no component of any $B_{i}$ is contained in any $B_{j}(j \neq i)$.

We shall deduce from (2) alone that at least $2 n-3$ of the sets $\operatorname{Fr}\left(B_{i}\right)$ $\cap \operatorname{Fr}\left(B_{j}\right)$ are nonempty; from (1) this will prove the theorem.

8.4. Lemma. For sets satisfying (2) above,

$$
\left[\operatorname{Fr}\left(B_{i}\right) \cap \operatorname{Fr}\left(B_{j}\right)=0\right] \leftrightarrows\left[\bar{B}_{i} \cap \bar{B}_{j}=0\right] .
$$

It will suffice to prove that if $\operatorname{Fr}\left(B_{i}\right) \cap \operatorname{Fr}\left(B_{j}\right)=0$ (for particular unequal values of $i$ and $j$ ) then $B_{i} \cap B_{j}=0$. Suppose the contrary; let $x \in B_{i} \cap B_{j}$, and let $C_{i}, C_{j}$ be the components of $B_{i}, B_{j}$ respectively which contain $x$. Then $C_{i}$ and $C_{j}$ are simple sets (cf. 3.1(1)) with disjoint frontiers; hence (from 4.5) either $C_{i} \cap C_{j}=0$, or $C_{i} \cup C_{j}=S$, or $C_{i} \supset C_{j}$, or $C_{j} \supset C_{i}$. The first alternative is excluded here (for $C_{i} \cap C_{j} \ni x$ ), and the others contradict (2) above.

8.5. Now form a linear graph $G$ having $n$ vertices $b_{1}, \cdots, b_{n}$, joining $b_{i}$ and $b_{j}$ (by a 1-cell) if and only if $\bar{B}_{i} \cap \bar{B}_{j} \neq 0$. Since $U \bar{B}_{i}=S$ (from 8.3(2)), we have

\section{(3) $G$ is connected.}

It may happen that a vertex of $G$, say $b_{1}$, has a shell which is not connected and which therefore falls in to two disjoint nonempty subgraphs, say $H$ and $K$. We may suppose the vertices of $H$ are $b_{2}, \cdots, b_{h}$ and those of $K$ are 
$b_{h+1}, \cdots, b_{k}$, where $2 \leqq h<k \leqq n$. Write $L=\bar{B}_{2} \cup \cdots \cup \bar{B}_{h}, M=\overline{B_{h+1}} \cup \cdots$ $\cup \bar{B}_{k}$; thus $L$ and $M$ are disjoint closed sets. Let $\left\{C_{\lambda}\right\}$ be the components of $B_{1}$. Then $\operatorname{Fr}\left(C_{\lambda}\right)$ is connected (3.2(ii)), and it is easy to see that $\operatorname{Fr}\left(C_{\lambda}\right) \subset L$ $\cup M$; hence, on defining $B^{\prime}=\bigcup\left\{C_{\lambda} \mid \operatorname{Fr}\left(C_{\lambda}\right) \subset L\right\}, B^{\prime \prime}=\bigcup\left\{C_{\lambda} \mid \operatorname{Fr}\left(C_{\lambda}\right) \subset M\right\}$, we have that $B^{\prime}, B^{\prime \prime}$ are nonseparating sets and that $B^{\prime} \cup B^{\prime \prime}=B_{1}$. Again, $B^{\prime}, B^{\prime \prime}$ are nonempty; for if $B^{\prime}=0$ we have $\operatorname{Fr}\left(B_{1}\right)=\operatorname{Fr}\left(B^{\prime \prime}\right) \subset M$ (on using $2.3(5)$ ), so that $\operatorname{Fr}\left(B_{1}\right) \cap \operatorname{Fr}\left(B_{2}\right)=0$, and therefore (8.4) $\bar{B}_{1} \cap \bar{B}_{2}=0$-contradicting the fact that $b_{2}$ belongs to the shell of $b_{1}$. Thus the sets $B^{\prime}, B^{\prime \prime}$, $B_{2}, B_{3}, \cdots, B_{n}$ have the same properties $(8.3(2))$ as the sets $B_{1}, \cdots, B_{n}$. Let $G_{1}$ be the linear graph formed from the sets $B^{\prime}, B^{\prime \prime}, B_{2}, \cdots, B_{n}$ in the same way as $G$ was formed from $B_{1}, \cdots, B_{n}$. Application of the lemma of 8.4 shows that $\bar{B}^{\prime} \cap \bar{B}^{\prime \prime}=0$, that $\bar{B}^{\prime} \cap \bar{B}_{i}=0$ unless $b_{i} \in H$, and that $\bar{B}^{\prime \prime} \cap \bar{B}_{j}$ $=0$ unless $b_{j} \in K$. Thus $\alpha_{1}\left(G_{1}\right) \leqq \alpha_{1}(G)$, and $\alpha_{0}\left(G_{1}\right)>\alpha_{0}(G)$. (Roughly speaking, $G_{1}$ is derived from $G$ by splitting the vertex $b_{1}$ into two vertices.)

If the vertices of $G_{1}$ do not all have connected shells, we repeat the argument; and so on, obtaining a sequence of graphs $G_{r}$ with $\alpha_{1}\left(G_{r}\right) \leqq \alpha_{1}\left(G_{r-1}\right)$ and $\alpha_{0}\left(G_{r}\right)>\alpha_{0}\left(G_{r-1}\right)$. But, as in (3) above, each $G_{r}$ is connected. Hence the process terminates, and we obtain a $G_{r}$ (taking $G=G_{0}$ ) all of whose shells are connected. The lemma of 8.2 then gives $\alpha_{1}(G) \geqq \alpha_{1}\left(G_{r}\right) \geqq 2 \alpha_{0}\left(G_{r}\right)-3 \geqq 2 \alpha_{0}(G)-3$. Thus there are at least $2 n-3$ pairs $(i, j)$, with $1 \leqq i<j \leqq n$, such that $\bar{B}_{i} \cap \bar{B}_{j}$ $\neq 0$; and, from 8.4 and $8.3(1)$, we have $\operatorname{Fr}\left(A_{i}\right) \cap \operatorname{Fr}\left(A_{j}\right) \neq 0$ for these pair The proof of Theorem 8 is thus complete.

8.6. Theorem 8 suggests the following more general problem: Given integers $h, k, n$, such that $2 \leqq h<k \leqq n$, and given $n$ connected sets such that every $h$ of them, but no $k$ of them, have a common point: how many pairs of their frontiers must intersect $\left({ }^{22}\right)$ ? The determination of the exact bound seems to be difficult (and probably not worth while); but it is easy to obtain a lower bound and an asymptotic formula. We need the following lemmas:

LEMMA 1. Let $A$ and $B$ be any connected sets, and let $\left\{C_{\lambda}\right\}$ be the collection of those components of $\operatorname{Co}(A)$ which meet $B$. Define $G=U \operatorname{Fr}\left(C_{\lambda}\right)$. Then $(A \cap B) \cup G$ is connected.

This is substantially the same as the lemma in 5.4 , and is proved by the same argument.

Lemma 2. Let $A_{1}, \cdots, A_{m}, B_{1}, \cdots, B_{n}$ be $m+n$ connected sets, where $m \geqq 2$ and $n \geqq 2$; and suppose that (i) every $A_{i}$ meets every $B_{j}(1 \leqq i \leqq m, 1 \leqq j \leqq n)$, (ii) $\cap A_{i}=0=\cap B_{j}$. Then, for at least one pair $(i, j), \operatorname{Fr}\left(A_{i}\right) \cap \operatorname{Fr}\left(B_{j}\right) \neq 0$.

Suppose not. Define $E_{1}, E_{2}, \cdots, E_{m}$ recursively by setting $E_{1}=A_{1}$ and $E_{i}=\left(A_{i} \cap E_{i-1}\right) \cup G_{i}$, where $G_{i}=\bigcup\left\{\operatorname{Fr}\left(C_{i \lambda}\right) \mid C_{i \lambda}\right.$ is a component of $\operatorname{Co}\left(A_{i}\right)$ meeting $\left.E_{i-1}\right\}$. Thus, from Lemma 1 , each $E_{i}$ is connected; and clearly

(22) The case $h=1$ is trivial, and so is excluded. 
$A_{1} \cap A_{2} \cap \cdots \cap A_{i} \subset E_{i} \subset\left(A_{1} \cap A_{2} \cap \cdots \cap A_{i}\right)$

$\cup \operatorname{Fr}\left(A_{2}\right) \cup \cdots \cup \operatorname{Fr}\left(A_{i}\right)$.

Now, each $B_{j}$ meets each $E_{i}$. For when $i=1$ this is given. Suppose that $B_{j}$ meets $E_{i-1}$. If $B_{j} \cap E_{i-1} \subset A_{i}, B_{j}$ meets $A_{i} \cap E_{i-1} \subset E_{i}$. In the remaining case, there exists a point $x \in B_{j} \cap E_{i-1} \cap D$, where $D$ is a component of $\operatorname{Co}\left(A_{i}\right)$. The connected set $B_{j}$ meets both $D$ and $\operatorname{Co}(D)$ (for $B_{j}$ meets $A_{i}$ ); hence $B_{j}$ meets $\operatorname{Fr}(D)$. But clearly $\operatorname{Fr}(D) \subset G_{i} \subset E_{i}$.

Thus, by induction, $B_{j}$ meets $E_{m}$, for every $j$. But $E_{m}$ is connected; and since $E_{m} \subset \operatorname{UFr}\left(A_{i}\right), E_{m} \cap \operatorname{Fr}\left(B_{j}\right)=0$. Hence $E_{m} \subset B_{j}$ for every $j$, contradicting $\cap B_{j}=0$.

REMARK. Since $\operatorname{Fr}\left(A_{1}\right)$ plays no part in this argument, there will be at least two pairs $i, j$ for which $\operatorname{Fr}\left(A_{i}\right) \cap \operatorname{Fr}\left(B_{j}\right) \neq 0$.

THEOREM 9. Let $A_{1}, \cdots, A_{n}$ be $n$ ( $\geqq 3$ ) connected sets, every two of which, but not all of which, have a common point. Let $h$ be the largest number such that every $h$ of them have a common point, and let $k$ be the smallest number such that no $k$ of them have a common point; thus $2 \leqq h<k \leqq n$. Then for at least $n(n-1)(2 h-1) / k(k-1)$ pairs $i, j$, with $1 \leqq i<j \leqq n, \operatorname{Fr}\left(A_{i}\right)$ meets $\operatorname{Fr}\left(A_{j}\right)$. Further, if $k$ is fixed and $n$ is large, then almost all the sets $\operatorname{Fr}\left(A_{i}\right) \cap \operatorname{Fr}\left(A_{j}\right)$ are nonempty. (More precisely, given $\epsilon>0$, there exists $N(\epsilon)$ such that if $n>\exp (k N(\epsilon))$ there are at most $\epsilon n^{2}$ pairs $i, j$ for which $\operatorname{Fr}\left(A_{i}\right) \cap \operatorname{Fr}\left(A_{j}\right)=0$.)

On applying Theorem 8 (8.1) we see that every $k$ of the sets $A_{i}$ must have at least $2 h-1$ pairs of intersecting frontiers among them; and a simple counting argument then gives the first part of the theorem. Suppose the second part is false, and form a linear graph $G$ having $n$ vertices $a_{i}$, in which $a_{i}, a_{j}$ are joined by a 1 -cell if and only if $\operatorname{Fr}\left(A_{i}\right) \cap \operatorname{Fr}\left(A_{j}\right) \neq 0$. Thus $\alpha_{1}(G)<(1 / 2-\epsilon) n^{2}$ and, if $n$ is large compared with $k$, a known theorem (see [11]) gives the existence of two groups of $k$ vertices of $G$, say $a_{1}, \cdots, a_{k}, b_{1}, \cdots, b_{k}$, such that no two vertices in different groups are adjacent. The corresponding sets, say $A_{1}, \cdots, A_{k}, B_{1}, \cdots, B_{k}$, violate Lemma 2 above, and the theorem is established.

\section{BIBLIOGRAPHY}

1. P. Alexandroff, Sur les multiplicites cantoriennes et le théorème de Phragmén-Brouwer generalise, C. R. Acad. Sci. Paris vol. 183 (1926) pp. 722-724.

2. P. Alexandroff and H. Hopf, Topologie I, Berlin, 1935.

3. R. E. Basye, Simply connected sets, Trans. Amer. Math. Soc. vol. 38 (1935) pp. 341-356.

4. K. Borsuk, Quelques théorèmes sur les ensembles unicohérents, Fund. Math. vol. 17 (1931) pp. 171-209.

5. - Über die Abbildungen der metrischen kompacten Räume auf die Kreislinie, Fund. Math. vol. 20 (1933) pp. 224-231.

6. — U Über den Lusternik-Schnirelmannsche Begriff der Kategorie, Fund. Math. vol. 26 (1936) pp. 123-136.

7. E. Čech, Sur les continus Péaniens unicoherrents, Fund. Math. vol. 20 (1933) pp. 232-243. 
8. S. Eilenberg, Transformations continues en circonference et la topologie du plan, Fund. Math. vol. 26 (1936) pp. 61-112.

9. - Sur les espaces multicohérents I, Fund. Math. vol. 27 (1936) pp. 153-190.

10. S. Eilenberg and E. W. Miller, Zero-dimensional families of sets, Bull. Amer. Math. Soc. vol. 47 (1941) pp. 921-923.

11. P. Erdös and A. H. Stone, On the structure of linear graphs, Bull. Amer. Math. Soc. vol. 52 (1946) pp. 1087-1091.

12. D. W. Hall and A. D. Wallace, Some invariants under monotone transformations, Bull. Amer. Math. Soc. vol. 45 (1939) pp. 294-295.

13. B. Knaster, Ein Zerlegungssatz über unikohärente Kontinua, Verhandlungen International Mathematische Kongress, Zurich, 1932 (2) p. 193.

14. B. Knaster and C. Kuratowski, Sur les ensembles connexes, Fund. Math. vol. 2 (1921) pp. 206-255.

15. C. Kuratowski, Sur les continus de Jordan et le theorème de M. Brouwer, Fund. Math. vol. 8 (1926) pp. 137-150.

16. - Une charactérisation topologique de la surface de la sphère. Fund. Math. vol. 13 (1929) pp. 307-318.

17. R. L. Moore, Foundations of point-set theory, Amer. Math. Soc. Colloquium Publications, vol. 13, 1932.

18. - Concerning upper semi-continuous collections of continua which do not separate a given continuum, Proc. Nat. Acad. Sci. U. S. A. vol. 10 (1924) pp. 356-360.

19. - Concerning intersecting continua, Proc. Nat. Acad. Sci. U. S. A. vol. 28 (1942) pp. 544-550.

20. M. H. A. Newman, Elements of the topology of plane sets of points, Cambridge University Press, 1939.

21. B. Pospísil, Trois notes sur les espaces abstraits, Publications de la Faculté des Sciences de l'Université Masaryk, No. 249, Brno, 1937.

22. M. Rueff, Über die Unikohärenz n-dimensionaler Polyeder, Comment. Math. Helv. vol. 7 (1934) pp. 14-23.

23. A. H. Stone, Parcompactness and product spaces, Bull. Amer. Math. Soc. vol. 54 (1948) pp. 977-982.

24. S. Straszewicz, Über die Zerschneidung der Ebene durch abgeschlossene Mengen, Fund. Math. vol. 7 (1925) pp. 159-187.

25. A. D. Wallace, Separation spaces, Ann. of Math. vol. 42 (1941) pp. 687-697.

26. - A characterization of unicoherence, Bull. Amer. Math. Soc. Abstract 48-11-345.

27. G. T. Whyburn, A junction property of locally connected sets, Bull. Amer. Math. Soc. Abstract 37-9-309.

28. — - Semi-closed sets and collections, Duke Math. J. vol. 2 (1936) pp. 685-690.

29. - Analytic topology, Amer. Math. Soc. Colloquium Publications, vol. 28, 1942.

30. R. L. Wilder, $A$ property which characterizes continuous curves, Proc. Nat. Acad. Sci. U. S. A. vol. 11 (1925) pp. 725-728.

31. W. A. Wilson, On the Phragmen-Brouwer theorem, Bull. Amer. Math. Soc. vol. 36 (1930) pp. 111-114.

33. G. S. Young, The introduction of local connectivity by change of topology, Amer. J. Math. vol. 68 (1946) pp. 479-494.

Trinity College, Cambridge University,

Cambridge, England. 